\title{
Proyecto de creación de un laboratorio de tutoría especializada en el desarrollo de competencias de emprendimiento en el Centro Universitario de Ciencias Bio- lógicas y Agropecuarias (CUCBA) de la Universidad de Guadalajara
}

\author{
${ }^{1}$ Oscar Carbajal Mariscal y ${ }^{2}$ Araceli Hernández Tinoco
}

\section{Project for the creation of a tutoring laboratory specialized in the de- velopment of entrepreneurship competencies at the University Cen- ter for Biological and Agricultural Sciences (CUCBA) of the Univer- sity of Guadalajara}

Profesor investigador, Departamento de Botánica y Zoología, Centro Universitario de Ciencias Biológicas y Agropecuarias de la Universidad de Guadalajara. ${ }^{2}$ Profesora investigadora, Departamento de Salud Pública, Centro Universitario de Ciencias Biológicas y Agropecuarias de la Universidad de Guadalajara.

autor de correspondencia: oscar. carbajal@academicos.udg.mx

\section{Resumen}

El campo laboral de los egresados del Centro Universitario de Ciencias Biológicas y Agropecuarias (CUCBA) de la Universidad de Guadalajara, se ha diversificado, debido a la disponibilidad de equipo tecnológico de alto nivel en diversas áreas del conocimiento científico. Por otra parte, el sector público mexicano, en estos últimos tiempos, ha restringido la contratación de varios perfiles profesionales, debido a las reducciones presupuestales para enfrentar la crisis energética en la que se sume nuestro país. Referente a la problemática de la inserción laboral, en la Universidad de Guadalajara se han realizado estudios de pertinencia de las diversas carreras. Una de las preocupaciones latentes de los organismos acreditadores de las licenciaturas es la forma cómo la universidad encara el futuro de los profesionales del CUCBA desde su formación académica en sus aulas. Todo lo anterior justifica la propuesta de creación de un Laboratorio de tutoría especializada en el desarrollo de competencias de emprendimiento del CUCBA, con la finalidad de apoyar al tutor de trayectoria para atraer a los jóvenes talentosos a las áreas que les representan oportunidades en el mercado profesional mexicano. La consolidación de esta nueva unidad departamental aprovecharía el contacto constante con los estudiantes del CUCBA para orientarlos en el aprendizaje para toda la vida y su consolidación como mexicanos con altos valores de solidaridad y conservación del medio ambiente con un enfoque sustentable.

Palabras clave: Tutoría académica de trayectoria, perfil profesional, inserción laboral, emprendimiento, mercado profesional mexicano, valores.

\footnotetext{
Abstract

The labor field of University Center for Biological and Agricultural Sciences (CUCBA) graduates at the University of Guadalajara, has diversified, due the external needs and the availability of diverse operational technological equipment in companies.
}

By the other hand, the Mexican public sector, in recent times, has restricted the hiring of several professional profiles, due to budget reductions to face the energy crisis in the country. About the problem of labor insertion, the University of Guadalajara has conducted studies of relevance of their diverse careers. One of the concerns latent certifiers from organisms is the way how the university faces the future of CUCBA professionals from its formation academic in their classrooms. All above, justifies the proposal for creation of tutoring laboratory specialized for development of entrepreneurship skills at CUCBA, with the purpose of supporting the career tutor to detect young talented people to the áreas that represent to them opportunities in the Mexican professional market. The consolidation of this new departmental unit would take advantage of constant contact with students of CUCBA to guide them in lifelong learning and their consolidation as Mexicans with high values of solidarity and conservation of the environment with a sustainable approach. Keywords: Academic tutoring of trajectory, professional profile, labor insertion, entrepreneurship, mexican professional market, values. 


\section{Antecedentes}

La economía global se encuentra en un estado de recesión que lleva acumulados varios años y las predicciones futuras no son optimistas en términos de estabilización y recuperación de los mercados mundiales, y lo más importante, de las oportunidades laborales. Por otra parte, la inclusión de tecnología de punta en la automatización de procesos industriales y el desarrollo de aplicaciones de Internet para atender demandas de servicios tradicionales, impactan negativamente en la disminución constante de determinadas oportunidades de trabajo.

Por mencionar algunos ejemplos del mercado laboral estadounidense, el empleo de programadores de computadoras, cuyo salario promedio anual es de $\$ 80,000$ USD, y que en 2014 cubría 329.000 puestos de empleo, hacia 2024 se tiene proyectada una oferta de empleo de tan sólo 302.000 lugares de trabajo lo cual representa una disminución de 27.000 puestos, es decir, una reducción equivalente al 8\%. Otros empleos que se ven amenazados, de acuerdo a esta proyección, son los relacionados con la operación de maquinaria -inclusive de maquinaria especializada-, los puestos de trabajo en actividades agrícolas: la atención de cultivos, viveros e invernaderos, el secretariado ejecutivo, entre otros. Contrasta el hecho de que el $75 \%$ de las ofertas de trabajo en los EE. UU requiera una educación universitaria. La educación formal es sólo una parte del proceso en los requisitos solicitados; los empleos de oportunidad también exigen más habilidades y experiencia. Por ejemplo, sólo el $27 \%$ de las ofertas de trabajo no piden requisitos específicos de experiencia, en comparación con el $64 \%$ de otras propuestas. ${ }^{1}$

Los resultados muestran que las oportunidades de trabajos no solo requieren habilidades más avanzadas en la manipulación de la

${ }^{1}$ https://www.weforum.org/agenda/2016/09/thesejobs-are-set-to-disappear-fastest-in-the-us?utm_content=buffera683f\&utm_medium=social\&utm_source=twitter.com\&utm_campaign=buffer tecnología y en la resolución de problemas, sino también exigen mayores habilidades sociales. El candidato ideal para una oportunidad de empleo debe estar familiarizado con el manejo del software pero también debe estar educado en un alto grado en el manejo de la inteligencia emocional.

En resumen, los empleadores están solicitando muchos requisitos y esto probablemente explica por qué los salarios han desafiado constantemente el estancamiento en determinadas ocupaciones. Para los solicitantes de empleo, lo anterior significa una búsqueda de superación constante y el aprendizaje formal y el cultivo de un conjunto más amplio de habilidades. Esta es una de las paradojas de la oportunidad: mientras que el $35 \%$ de las ofertas de trabajo son para los empleos mejor pagados, sólo el $27 \%$ de todos los clics van en busca de ellas. A pesar de que pagan mejor, la brecha de talento persiste. Para los empleadores, sin embargo, significa que dichos puestos de trabajo son difíciles de llenar. ${ }^{2}$

En México, en el panorama para 2015 que reporta la empresa Hays (Recruiting experts worldwide) se insiste en la inversión en la capacitación de los trabajadores, para evitar el reciclado del talento sin nuevas competencias laborales. En el mencionado reporte se destaca el hecho de que:

"muchos empleados ya no quieren hacer carrera permanente en una empresa y los más calificados se han vuelto más selectivos. Los empleadores necesitan cambiar de visión, pero a muchos les da miedo tomar decisiones dramáticas, siguen siendo conservadores." ${ }^{3}$

Al igual que en el paradigma estadounidense, las novedosas tecnologías exigen una mayor capacitación y por ende, mayores sala-

${ }^{2}$ http://blog.indeed.com/wp-content/uploads/2016/07/State_of_Opportunity-Indeed_Hiring_Lab.pdf

${ }^{3}$ http://www.hays.com.mx/cs/groups/hays_common/@ mx/@content/documents/digitalasset/hays_1419791.pdf 
rios, pero tanto la inversión en tecnología como en salarios ha frenado dicha adopción en muchas empresas mexicanas.

Se destaca en dicho informe que habilidades tan relevantes como el dominio del idioma inglés, el trabajo colaborativo, el liderazgo y las capacidades de manejo presupuestal, están ausentes en los profesionales que llenan las solicitudes de empleo.

"Por otro lado, la educación en México se mantiene como un tema de dogma, donde se respeta a los sindicatos y, en consecuencia, son ellos los que están formando a las generaciones de trabajadores." 4

Una posibilidad para el escenario mexicano en el futuro próximo, será la polarización de la Población Economicamente Activa: se insiste que las funciones de baja especialidad se quedarán de muy baja especialidad, las de media, terminarán por desaparecer, y las altas se volverán más caras, con elevados costos para la competitividad en la industria y el sector de servicios mexicanos.

"Pero hay una solución si se concreta la colaboración entre las industrias, los gobiernos y el sector educativo para formar las habilidades requeridas. Ello implica poner en marcha los cursos, el aprendizaje y la formación necesaria, proporcionando además un financiamiento adecuado.

Las empresas seguirán teniendo un papel fundamental porque son las encargadas de crear una cultura del valor, para que el empleador sepa gestionar las diferentes competencias de cada uno de sus trabajadores y ayude a que se autodesarrollen." 5

En los últimos años, las Instituciones de Educación Superior (IES) de México han realizado actividades colegiadas, tendientes a la ade-

\footnotetext{
${ }^{4}$ Idem.

${ }^{5}$ Idem.
}

e.cucba / ENERO-JUNIO/ AÑO 4/ NÚMERO 7/1-11 cuación de sus planes de estudio, acordes a las necesidades del mercado laboral. En el Plan Nacional de Desarrollo 2013-2018 presentado por el actual ejecutivo, se establece en el capítulo Un México con Educación de Calidad, que las medidas a implementar en este rubro, servirán

"para garantizar un desarrollo integral de todos los mexicanos y así contar con un capital humano preparado, que sea fuente de innovación y lleve a todos los estudiantes a su mayor potencial humano.

El enfoque, en este sentido, será promover políticas que cierren la brecha entre lo que se enseña en las escuelas y las habilidades que el mundo de hoy demanda desarrollar para un aprendizaje a lo largo de la vida." ${ }^{6}$

El énfasis en la innovación y en el aprendizaje para la vida nos remite a la intención de despertar en la población mexicana la necesidad de desarrollar sus habilidades innatas y descubrir áreas de influencia en las cuales dicho talento pueda invertirse ${ }^{7}$. Entre las estrategias del mencionado Plan Nacional de Desarrollo, tenemos:

"Estrategia 3.1.3. Garantizar que los planes y programas de estudio sean pertinentes y contribuyan a que los estudiantes puedan avanzar exitosamente en su trayectoria educativa, al tiempo que desarrollen aprendizajes significativos y competencias que les sirvan a lo largo de la vida.

Estrategia 3.1.5. Disminuir el abandono escolar, mejorar la eficiencia terminal en cada

${ }^{6}$ Plan Nacional de Desarrollo 2013-2018

7“Una elevada proporción de jóvenes percibe que la educación no les proporciona habilidades, competencias y capacidades para una inserción y desempeño laboral exitosos. En línea con esta preocupación, el 18\% de los participantes en la Consulta Ciudadana opinó que para alcanzar la cobertura universal, con pertinencia en educación media superior y superior, se deben fortalecer las carreras de corte tecnológico y vincularlas al sector productivo." (Plan Nacional de Desarrollo 2013-2018) 
nivel educativo y aumentar las tasas de transición entre un nivel y otro." 8

Uno de los objetivos de la tutoría académica, es disminuir el rezago y minimizar la deserción, los cuales son amenazas potenciales que se presentan a lo largo de la trayectoria de los estudiantes. De ahí que se lleven a cabo diversas acciones orientadas a responder a esta problemática, desde la búsqueda de estrategias innovadoras para la tutoría, hasta la capacitación y sensibilización de los profesores tutores en los diversos ámbitos del desarrollo humano, el manejo de las emociones, el diseño y seguimiento de los planes y proyectos de vida, entre otros.

\section{Justificación}

El Rector de la Universidad de Guadalajara, Mtro. Itzcóatl Tonatiuh Bravo Padilla, relató en su informe de actividades de 2015, la importancia de medir y reducir la huella ecológica que generan los miembros de la Red Universitaria, con la finalidad de

“...reconocer el desafío de permear esta visión en aplicaciones innovadoras del conocimiento y la tecnología, así como en el fortalecimiento de las actividades docentes, de investigación, y de extensión con visión de sostenibilidad."

El Centro Universitario de Ciencias Biológicas y Agropecuarias es una institución altamente comprometida con las acciones anteriores, y dada la naturaleza de sus ejes temáticos, es prioritario el enfoque emprendedor tanto de profesores como de alumnos, quienes tienen en sus manos la oportunidad de innovar y acceder a fuentes de recursos materiales y de capital humano, comprometido con la conservación del medio ambiente.

\footnotetext{
${ }^{8}$ Idem.
}

${ }^{9}$ http://www.rectoria.udg.mx/sites/default/files/ IA2015-mensajeTBP.pdf
En el mismo informe, el Mtro. Itzcóatl Tonatiuh Bravo Padilla hace hincapié en la participación activa que los miembros de la Red Universitaria tuvieron en

"distintos encuentros internacionales vinculados a la innovación y al emprendimiento realizados en Jalisco.

A mediados del año, la Universidad de Guadalajara coorganizó Campus Party, encuentro en el que se presentaron temas de vanguardia en tecnología, emprendimiento y creatividad, contando con la asistencia de estudiantes y académicos de la Red Universitaria quienes participaron a través de conferencias y talleres. Esta colaboración se extendió para coorganizar "Campus Night", donde las distintas industrias relacionadas con la tecnología debatieron sobre innovación y las nuevas tendencias.

La institución también otorgó 550 becas para asistir al encuentro Wobi On Entrepreneurship 2015, evento donde asistieron 4 mil emprendedores a las múltiples conferencias con invitados de clase mundial que se dieron cita." 10

Las actividades mencionadas establecen un interés constante en la vinculación con las fuentes externas de financiamiento, la participación en ferias y encuentros de innovación y emprendimiento.

En el Centro Universitario de Ciencias Económico Administrativas (CUCEA) de la Universidad de Guadalajara, en colaboración con la Universidad de Virginia Commonwealth, se creó el Centro Internacional de Excelencia Empresarial (CIEE)

"con el fin de contribuir al desarrollo de negocios con la prestación de servicios de capacitación, consultoría, incubación e información para que puedan obtener mejores resultados en el actual contexto de globalización e integración internacional.

\footnotetext{
${ }^{10}$ Idem.
} 
La experiencia, el profesionalismo y la investigación aplicada de estas universidades representan una plataforma para el desarrollo de proyectos innovadores, y fortalecen con una visión a largo plazo los esfuerzos de profesionalización y desarrollo de organismos públicos y privados." 11

El objetivo de este Centro Internacional de Excelencia Empresarial es:

"Incentivar la vinculación empresa-universidad-gobierno, equilibrando los intereses de todos los actores (estudiantes, académicos, empresarios, gobierno, personal administrativo y público en general), generando así beneficios para la sociedad por medio de la creación de empleos y de nuevas empresas." 12

El CIEE empieza a tener una presencia importante en el CUCBA, al organizar eventos de difusión sobre los servicios que presta a la comunidad universitaria, así como el contacto permanente con los estudiantes de las carreras de Agronegocios y Ciencia de los Alimentos que están estrechamente vinculados con las labores de tipo emprendedor.

\section{Fundamentación en el marco legal universi- tario}

En la Ley Orgánica de la Universidad de Guadalajara se establecen los fundamentos para la creación del Laboratorio de tutoría especializada en el desarrollo de competencias de emprendimiento:

"Artículo 52. Son atribuciones de los Consejos de Centros Universitarios:

...

Proponer al Consejo General la creación, modificación, o supresión de dependencias y programas del Centro, de acuerdo a la normatividad general vigente en la Universidad;

\footnotetext{
${ }^{11}$ http://ciee.cucea.udg.mx/?q=ciee/antecedentes-ciee

${ }^{12}$ Idem.
}

Q-cucba / ENERO-JUNIO/ AÑo 4/ NÚMERO 7/1-11
Artículo 65. Las atribuciones de los Colegios Departamentales, en relación a las actividades de sus respectivos departamentos, serán las siguientes:

...

Proponer al Consejo Divisional la creación, supresión o modificación de los departamentos y sus unidades;" 13

Por su parte el Estatuto General de la Universidad de Guadalajara en su Artículo primero, establece que:

"El Estatuto General ... regula la integración, estructura, organización y funcionamiento de la Red Universitaria en el Estado de Jalisco." 14

La creación del Laboratorio de tutoría especializada en el desarrollo de competencias de emprendimiento se particulariza en los artículos 130 y 160 :

"Artículo 13. Para el desempeño de sus funciones, los Departamentos se integrarán a partir de unidades académicas, en cualquiera de las siguientes cuatro modalidades, que serán las siguientes:

I.-Institutos;

II. Centros de investigación;

III. Laboratorios, y

IV. Academias.

La organización de estas unidades y el procedimiento para la designación de sus Titulares, se normará por el Estatuto Orgánico respectivo. La propuesta para su constitución deberá contar, en todo caso, con la autorización del Colegio Departamental que corresponda.

Artículo 16. Se define como Laboratorio a la unidad departamental que realiza funciones de apoyo a la investigación, docencia o difusión. En todo caso, deberá cumplir los si-

\footnotetext{
${ }^{13}$ Ley Orgánica de la Universidad de Guadalajara http://www. secgral.udg.mx/sites/archivos/normatividad/general/Leyorganica.pdf

${ }^{14}$ Estatuto General de la Universidad de Guadalajara 2016 http://secgral.udg.mx/sites/archivos/normatividad/gene-
} 
guientes requisitos:

I.-Contar con una plantilla académica, de cuyos miembros uno al menos, tenga la categoría de asociado;

II. Cumplir funciones especializadas de apoyo al Departamento en forma sistemática;

III. Contar con instrumentos de planeación, programación, presupuestación y evaluación de sus programas; $\mathrm{y}$

IV. Contar con los recursos financieros necesarios para su funcionamiento." 15

\section{Descripción de la propuesta: Creación de un Laboratorio de tutoría especializada en el de- sarrollo de competencias de emprendimiento.}

En la página oficial del CUCBA se establece, en su misión, que:

"El Centro Universitario de Ciencias Biológicas y Agropecuarias de la Universidad de Guadalajara, es una comunidad académica, dedicada a la formación de recursos humanos de calidad en ciencias biológicas, agropecuarias y ambientales con compromiso social; realiza investigación científica y desarrollo tecnológico, con énfasis en la comprensión de los procesos biológicos, el manejo y aprovechamiento de los recursos naturales, la producción agropecuaria, la salud pública, y la sanidad animal, vegetal y ambiental como contribución al desarrollo sustentable, con impacto nacional e internacional." 16

Desde su formación en 1993, el CUCBA forma parte de la Red Universitaria de Jalisco. En la actualidad, atiende una población estudiantil de 5300 estudiantes -en aumento semestre a semestre- en las carreras de:

- Licenciatura en Biología

- Lic. en Ing. Agrónomo

- Lic. en Ciencia de los Alimentos

- Lic. en Agronegocios

- Lic. en Medicina Veterinaria y Zootecnia.

ral/EG\%20dict\%20088.2016.pdf

${ }^{15}$ Idem.

${ }^{16}$ http://www.cucba.udg.mx/acerca-de/mision-y-vision
Además de contar con 8 programas de posgrado, uno de ellos totalmente en línea.

La propuesta de creación de una unidad departamental denominada Laboratorio de tutoría especializada en el desarrollo de competencias de emprendimiento, pretende cubrir las necesidades existentes en este ámbito en el acompañamiento de alumnos y profesores del CUCBA, a través de diversas actividades de tipo transversal y multidisciplinar. Tales necesidades se expresan en el análisis FODA, de la propia institución, en el rubro Debilidades:

"4. Programa de tutorías ineficaz.

...

6. Coordinación y seguimiento de la atención integral a la comunidad del CUCBA." ${ }^{17}$

La propuesta responde a una necesidad real del CUCBA por contar con metodologías y estrategias que propicien el emprendimiento en el personal docente y en los alumnos. Debido a que no se dispone del personal académico con experiencia en emprendimiento, tampoco se desarrollan dichos valores entre los alumnos del Centro Universitario.

Las competencias emprendedoras no son una actividad común en el CUCBA. Algunas veces el emprendimiento crea inseguridad o se cree que se refiere a otras personas y no para toda la comunidad universitaria. El desarrollo de las competencias emprendedoras es incluyente. Mediante la promoción, capacitación y generación de proyectos se establecerá la confianza entre los alumnos y maestros, para convertir al emprendimiento en una parte sustancial de los valores del capital humano del Centro Universitario.

El Laboratorio de tutoría especializada en el desarrollo de competencias de emprendimiento, al estar vinculado con las diversas instancias de la Red Universitaria, y a los pro-

\footnotetext{
${ }^{17}$ Idem.
} 
gramas de apoyo financiero a la innovación emprendedora del municipio, del estado de Jalisco, del gobierno federal y de la iniciativa privada, dispondría de recursos financieros suficientes para apoyar las propuestas individuales de los estudiantes, en la búsqueda del beneficio social.

\section{Objetivo:}

El Laboratorio de tutoría especializada en el desarrollo de competencias de emprendimiento tiene la finalidad de acompañar y orientar a los estudiantes del Centro Universitario de Ciencias Biológicas y Agropecuarias de la Universidad de Guadalajara en el conocimiento de las innovaciones referentes a los ejes de desarrollo del CUCBA y en la mejora de sus habilidades emprendedoras, considerando una actitud abierta y propositiva para la toma de decisiones, así como los valores de trabajo en equipo, equidad, solidaridad, respeto por la propiedad intelectual y el desarrollo sustentable.

\section{Departamento receptor:}

El Departamento de Botánica y Zoología es una entidad de la División de Ciencias Biológicas y Ambientales. Se caracteriza por desarrollar investigación básica y aplicada de alto nivel, además de contar con el Herbario IBUG, uno de los acervos de plantas más grandes e importantes del Occidente de México. A través de proyectos transversales y multidisciplinares, este Departamento se vincula con otras entidades del propio CUCBA, así como nacionales e internacionales, en redes de cooperación académica. Esta vinculación con otras entidades caracteriza y ha caracterizado el espíritu de emprendimiento de los académicos y directivos del Departamento de Botánica y Zoología, en la búsqueda de nuevas formas de colaboración en proyectos de beneficio común.

\section{Establecimiento en el organigrama:}

El laboratorio de tutoría especializada en el desarrollo de competencias de emprendimiento se situaría en el organigrama del Centro
Universitario de Ciencias Biológicas y Agropecuarias, tal como se describe en la Fig. 1:

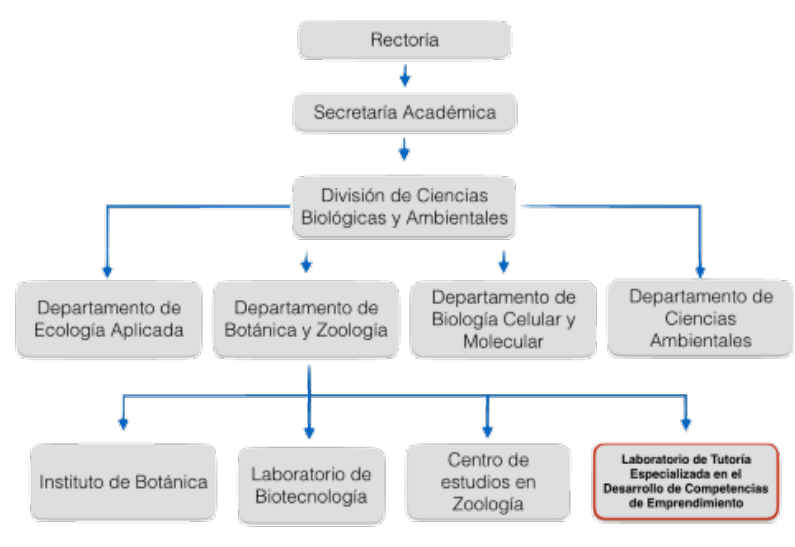

Fig. 1 Localización del Laboratorio de Tutoría en el organigrama del CUCBA. Gráfico de autoría propia.

\section{Profesores investigadores fundadores de la nueva Unidad Departamental}

Dra. Araceli Hernández Tinoco. Es Profesor Docente Titular A, adscrita al Departamento de Salud Pública. Cuenta con experiencia en la creación de empresas PyME y de tramitología en Secretaría de Promoción económica CEPE y Secretaría de Economía SE. Fue beneficiaria de proyectos de apoyo Federal y Estatal para proyectos de innovación PyME por CEPE y FOJAL. Cuenta con capacitación en el área de emprendimiento Social y creación de nuevas empresas. Dos veces beneficiaria de incubación en Tec de Monterrey, Campus Guadalajara para proyectos PyME. Participante de la primera generación de formadores de emprendimiento de la universidad y participante del primer Symposium for Entrepreneurship Educators SEE Program for México por el Babson College, la escuela de negocios y emprendimiento más importante del Mundo.

M. en B. A. Oscar Carbajal Mariscal. Es Profesor Investigador Asociado A, adscrito 
al Departamento de Botánica y Zoología. Es experto en el diseño instruccional de cursos en línea y de actividades de aprendizaje para modalidades presenciales y virtuales. Ha sido beneficiado con beca por parte del Consejo Estatal de Creación Artística (CECA) para el desarrollo de producción audiovisual. Es miembro fundador de la Red de Comunicación y Divulgación de la Ciencia de la Universidad de Guadalajara, y es miembro suplente del Comité para la Innovación en Ambientes de Aprendizaje.

\section{Estrategias de tutoría para el desarrollo de competencias de emprendimiento:}

Primordialmente, las estrategias de tutoría están enfocadas en la localización del talento humano. Se infiere que, a partir de los valores que el ser humano ha arraigado con profundidad en su diario vivir, se desarrollan las actitudes que favorecen la significación de nuevos conocimientos $\mathrm{y}$, por ende, de nuevas habilidades.

Oportunidades para la detección del talento humano con habilidades emprendedoras en el CUCBA

A continuación se describen los momentos de alta motivación por parte de los estudiantes en los que ocurre tal detección por parte del tutor de trayectoria:

1. En las carreras del CUCBA las prácticas de laboratorio y de campo forman parte de sus actividades académicas y en estos procesos los jóvenes descubren y revelan los intereses y las actitudes que muchas veces detonan en la orientación vocacional y el proyecto de vida.

2. A través de las estrategias didácticas como el aula invertida (flipped class), los jóvenes experimentan una nueva forma de aprender, asumen responsabilidades y desarrollan productos de forma colaborativa.

3. Actividades de Trabajo Colabora- tivo. En esta estrategia se desarrollan habilidades para realizar el trabajo en equipo. Los resultados en la detección del talento humano se dan tanto en la forma presencial como en línea.

4. Participación en foros científicos: Semana de la Investigación. En el CUCBA se celebra año con año la Semana de la Investigación, la cual cumple 19 años de ejecución. En este foro de investigación participan todo tipo de estudiantes, no sólo los tesistas, quienes han contribuido en la investigación con diversas actividades. En estos foros, al igual que en la participación de conferencias magistrales se descubre el talento humano y su futura trayectoria profesional y de vida.

5. Las Jornadas de Biología (JOBIs) y la Semana de la Superación Agronómica. En los meses de marzo-abril de cada año tiene lugar una semana académica para los estudiantes de Biología, Agronomía y Agronegocios en la que se imparten diversos talleres, cursos y conferencias. En esas jornadas, de nueva cuenta, aflora el talento humano y la vinculación temprana del estudiante en la investigación, la docencia y la difusión de la cultura, así como con actividades emprendedoras de innovación.

6. Prácticas profesionales $y$ servicio social. En las distintas áreas del CUCBA los estudiantes han cursado sus prácticas y servicio social. Algunos de ellos, por ejemplo, han encontrado en el manejo de las Tecnologías de la Información y Comunicación y el diseño y elaboración de materiales didácticos y de difusión científica, una veta profesional en la que se han desempeñado en las etapas 
posteriores de su vida.

7. Seminarios de fin de curso de Ciencia de los Alimentos. En estos seminarios, los alumnos de la carrera exponen los avances de sus trabajos de diseño y creación de nuevas opciones de alimentos. Muchas de esas opciones son innovadoras y representan verdaderas oportunidades de negocio.

8. ExpoEmprendedores del CUCBA. Esta Exposición que se acerca a su 12a. edición ha sido un foro de exhibición del espíritu innovador y emprendedor del estudiantado del CUCBA quienes han desarrollado su proyecto particular de emprendimiento de un negocio, a partir de los conocimientos adquiridos en las aulas de nuestra institución.

\section{Otras estrategias de docencia, investigación y vinculación:}

Consolidar un Diplomado permanente en el desarrollo de competencias de emprendimiento.

Inculcar en los estudiantes y profesores el concepto de emprendimiento mediante experiencias personales directas, formación de grupos multidisciplinarios desde el inicio de la formación de las propuestas innovadoras, en los que se incluya a estudiantes de otros Centros Universitarios de la Red, realización de eventos transdisciplinares, implementación de estrategias de comunicación y de capacitación de profesores para la implementación de actividades de emprendimiento en clase.

Promover el cambio al emprendimiento en el Centro Universitario mediante concursos y aplicación de proyectos de emprendimiento surgidos en el CUCBA para la solución de problemáticas internas, a través de soluciones generadas por estudiantes y profesores relacionadas con los diferentes ejes de conocimiento del CUCBA.

Crear vinculación con las incubadoras y aceleradoras de la Red Universitaria, aprovechando los recursos y la expertis de los académicos profesionales en este ámbito, vinculando al Laboratorio de tutoría especializada en el desarrollo de competencias emprendedoras del CUCBA con el Centro Empresarial para la Calidad del Centro Universitario de los Lagos (CULAGOS) y el Centro Internacional de Excelencia Empresarial de CUCEA.

Promover programas de captación de proyectos potenciales de emprendimiento para darles seguimiento y apoyo derivándolos a las siguientes etapas de incubación, acceso a recursos y puesta en marcha de CUCEA y CULAGOS.

Obtener recursos financieros externos a través de la vinculación constante con organismos de la Red Universitaria de Jalisco, relacionada con las competencias emprendedoras, para fortalecer esta visión conjunta y ofrecer constantemente la oportunidad de generar proyectos y asesorar a los autores en su trayectoria hasta la implementación.

Generar la confianza del emprendimiento con el apoyo de un organismo estructurado formado por elementos profesionales competentes y con amplia experiencia de trabajo en el área para brindar asesorías personalizadas de alto nivel.

Realizar eventos y exposiciones con casos de éxito para que los emprendedores compartan sus experiencias con alumnos y profesores.

Conducir mesas de trabajo y/o talleres para lluvias de ideas y simulacros de generación de ideas de productos y servicios potenciales para nuevos negocios para los estudiantes de las 
carreras de Biología, Veterinaria, Agronomía, Ciencia de los alimentos y Agronegocios.

\section{Proyectos especiales}

Una cualidad de las iniciativas emprendedoras es su constante innovación. A continuación, se describen algunas estrategias para implementar:

Se pretende normar la creación y gestión de un Diplomado permanente en el desarrollo de competencias de emprendimiento a través de un Mooc (Massive open on-line course), gestionado e impartido por el personal altamente especializado del Laboratorio de tutoría especializada en el desarrollo de competencias de emprendimiento.

Crear un Centro de Información en el que se capten las necesidades potenciales en emprendimiento de zonas, regiones, áreas y grupos sociales, con quienes se pueda interactuar en línea, con el fin de generar y brindar soluciones factibles a las ideas de negocio para su consolidación en el futuro.

Promover concursos para la solución de problemas específicos de vinculación Universidad-Empresa que se puedan ofrecer como servicio a la comunidad, con el compromiso de ofrecer empleo a los alumnos que desarrollen la propuesta ganadora de la empresa objetivo, con el fin de ingresar recursos financieros a la Unidad Departamental.

Generar con los grupos de alumnos de Servicio social pequeñas campañas de apoyo a la comunidad mediante el ofrecimiento de servicios que surgieron de proyectos de emprendimiento relacionados con sus áreas de especialidad (biología, agricultura y agronegocios, ciencia de los alimentos y medicina veterinaria). Que desarrollen actividades y servicios relacionados con su capacitación básica de carrera cualquiera que sea.
Ejemplo de caso de tutoría especializada en emprendimiento: El conocimiento de la Naturaleza y los valores.

En un breve curso taller sobre la Elaboración de microdosis a partir de extractos de plantas medicinales que se impartió a los estudiantes de la Lic. en Biología del CUCBA en 2015, se detectó que el interés de los jóvenes por tomar dicho curso consistía en "aprovechar racionalmente los recursos naturales mexicanos en el beneficio de la salud de la sociedad", lo cual se traduce en dos valores primordiales: la solidaridad y el desarrollo sustentable. Ambos valores, vinculados con los conocimientos previos de los estudiantes más o menos amplios de $t a-$ xonomía, biología celular, biología molecular, botánica, inmunología, entre otras asignaturas, y el interés por aprender a elaborar los extractos vegetales, les proporcionó las bases para desarrollarse en el conocimiento de la etnobotánica y el manejo de la fitoterapia. Se presentaron dos casos de estudiantes, quienes revelaron su talento emprendedor: decidieron continuar su preparación integral (especialización en los procesos industriales para la obtención de los extractos vegetales, propiedad intelectual y marketing, en una primera etapa) para crear su propia línea de productos de origen natural para la salud y la belleza que les ayudara a obtener beneficio económico, enfocándose en los valores éticos mencionados con anterioridad.

\section{Conclusiones}

El talento humano con habilidades emprendedoras -en las aulas del Centro Universitario- se puede detectar e incentivar a través de diversas actividades. La creación del Laboratorio de tutoría especializada en el desarrollo de competencias de emprendimiento del CUCBA, promueve al tutor de trayectoria para atraer a los jóvenes talentosos a las áreas que les representan oportunidades en el vasto mercado profesional mexicano: los presenta con los académicos que realizan investigación afín a sus intereses; los relaciona con las distintas opciones de inves- 
tigación, docencia y extensión, y los vincula con las instancias que aportan apoyos privados y estatales dirigidos a la creación de iniciativas emprendedoras, incluidas las de negocio. La consolidación de esta nueva unidad departamental aprovecharía el contacto constante que tienen los estudiantes del CUCBA con tales oportunidades, para orientarlos en el aprendizaje para toda la vida y su consolidación como mexicanos con altos valores de solidaridad y conservación del medio ambiente con un enfoque sustentable

\section{Literatura citada}

https://www.weforum.org/agenda/2016/09/these-jobsare-set-to-disappear-fastest-in-the-us?utm_content $=$ buffera683f\&utm_medium $=$ social \&utm_source=twitter.com\&utm_campaign=buffer
http://blog.indeed.com/wp-content/uploads/2016/07/State_of_Opportunity-Indeed_Hiring_Lab.pdf

http://www.educacionyculturaaz.com/educacion/la-educacion-superior-y-el-mercado-de-trabajo-en-mexico

$\mathrm{http} / / /$ ciee.cucea.udg.mx/?q=ciee/antecedentes-ciee http://ciee.cucea.udg.mx/?q=ciee/objetivo

http://www.cucba.udg.mx/acerca-de/mision-y-visio Estatuto General de la Universidad de Guadalajara 2016

http://secgral.udg.mx/sites/archivos/normatividad/gene$\mathrm{ral} / \mathrm{EG} \% 20 \mathrm{dict} \% 20088.2016 . p d f$ Ley Orgánica de la Universidad de Guadalajara

http://www.secgral.udg.mx/sites/archivos/normatividad/ general/Leyorganica.pdf

Mercado laboral mexicano 2015

http://www.hays.com.mx/cs/groups/hays_common/@ mx/@content/documents/digitalasset/hays_1419791. pdf 